\title{
Discursive Practices
}

of Intercultural Paternalism

in Modern Migration Societies

in the German-Speaking Area

\author{
Lyudmila V. Kulikova* \\ Siberian Federal University \\ 79 Svobodny, Krasnoyarsk, 660041, Russia
}

Received 02.12.2016, received in revised form 15.12.2016, accepted 11.01.2017

\begin{abstract}
The paper deals with the concept of intercultural paternalism and its discursive realization in the multi-cultural context of modern migration societies (on the communicative-linguistic material of the German-speaking area).

On the basis of theoretical generalizations and collected empirical data the author considers the possibility of classifying the discursive practices of paternalism in correlation with institutional and non-institutional spheres of its existence, pragmatics of intercultural situations and differentiation of the communicants' role positions. The study reveals the essence of discourse processing of the paternalistic actions and, on the example of the author's cases, the means of discourse processing in the situations of interaction of the actants with different cultural conventionality are described in detail. The attention is focused on the need to change the behavioral patterns in power structures, migration services, educational and social organizations in the conditions of new social realities.

The main methods of the conducted mini-research are overt observation, interview, case-method, study of the specialized scientific literature and German-language press publications, media space monitoring.
\end{abstract}

Keywords: paternalistic discourse in multi-cultural societies, forms and contexts of paternalism, discourse processing of paternalistic practices, stages of discourse processing.

DOI: 10.17516/1997-1370-0005.

Research area: philology.

(C) Siberian Federal University. All rights reserved

* Corresponding author E-mail address: info_ifiyak@sfu-kras.ru 


\title{
Diskursive Praktiken
}

\section{des interkulturellen Paternalismus \\ in den modernen Migrationsgesellschaften \\ (am Beispiel des deutschsprachigen Raums)}

\author{
Ljudmila W. Kulikowa \\ Sibirische Föderale Universität \\ 79 Swobodnyj, Krasnoyarsk, 660041, Russia
}

\begin{abstract}
Der vorliegende Beitrag, der dem Thema des interkulturellen Paternalismus gewidmet ist, hat folgende Ziele:

- die kritische Auseinandersetzung mit dem Begriff Paternalismus und seiner Begriffsentwicklung in der modernen Integrationsdebatte;

- die Erläuterung der diskursiv-kommunikativen Dimension des Phänomens Paternalismus;

- die Analyse der Formen der paternalistischen Beziehungen in den Situationen der interkulturell bedingten Kommunikation.

Im theoretischen Input des Beitrags wird Paternalismus als diskursives Phänomen modelliert, mit Bezug auf Prozesse der Ausarbeitung und Behandlung der kommunikativen Irrationalität des kulturell Anderen, die paternalistische Eingriffe verursacht. Es werden auch Kontexte (institutionell, alltäglich, staatlich, privat) und Formen/Typen des interkulturellen Paternalismus behandelt.

Wie die empirische Untersuchung zeigt, führen Kontaktsituationen zwischen Inländern und Ausländern praktisch ohne Ausnahme in verschiedenen Verhaltensbereichen zu Disparitäten und Irritationen. Anhand von Fallbeispielen versucht der Autor aus der kulturellen und diskursanalytischen Perspektive paternalistische Interaktionsmuster zu problematisieren. Die Frage, inwieweit die kultursensible sowie effektive gleichwertige Kommunikation mit der Migrantenklientel als «Gegendiskurs» zu Praktiken der Entmündung und Herabsetzung gesehen wird, soll Ausgangspunkt für die abschließende Diskussion sein.
\end{abstract}

Ключевые слова: paternalistischer Diskurs in der Migrationsgesellschaft, Kontexte und Formen des interkulturellen Paternalismus, diskursive Bearbeitung der paternalistischen Praktiken, sequenzielle Struktur des Paternalismus.

\section{Zur Einführung}

Die Debatte um Zuwanderung und Integration ist derzeit eine der zentralen gesellschaftlichen Auseinandersetzungen in Europa. Dabei betrifft dieses Thema auch die paternalistische Figuration, die sowohl die sozial wirksame Differenz zwischen Gruppen „mit und ohne Migrationshintergrund“, als auch die interkulturell bedingte Kommunikation zwischen Inländern und Ausländern markiert und die im amtlichen Raum sowie im Alltag die Interaktionsmuster und Handlungsweisen beeinflusst (dabei handelt es sich oft um bestimmte Subtilitäten in den interkulturellen Beziehungen). Unter Paternalismus versteht man bekanntlich im allgemeinen Bevormundung, den sog. Paternalismus alter Prägung (Dvorkin, 2005; Aaken van, 2006; Heiko, 2006) und zudem noch die besondere Art der Machtbeziehungen. Darüber hinaus haben paternalistische Praktiken und Diskurse als Widerspiegelung des Herrschaftsverhältnisses zu tun mit solchen Konstellationen wie Macht, Dominanz, Status, Asymmetrie, hilfsbedürftiges Personenprofil der Migrant_innen oder Ausländer_innen etc. 
Das Ziel dieses Beitrags ist es, anhand des vorhandenen theoretischen und praktischen Wissens Paternalismus $\mathrm{zu}$ beschreiben als kommunikatives Phänomen im Bereich der neuen interkulturellen Realität.

\section{Methoden und theoretischer Rahmen}

Die Daten, die in dieser Untersuchung analysiert und systematisiert werden, sind hauptsächlich aus Beobachtungen des Alltagslebens (auch aus der ethischen Perspektive des Forschers als professioneller Fremder), durch Interviews und schriftliche Quellen erhoben worden. Auch Befragungen und Erlebnisse (Fälle) der Betroffenen wurden berücksichtigt. Der empirischen Untersuchung im Feld ging selbstverständlich eine Recherchephase der fachliterarischen Landschaft voraus.

Als Einstieg ins Thema erlaube ich mir vor allem die für den Beitrag wichtigsten Termini und Studien zu erwähnen.

MitdemBegriff,,Paternalismus“bezeichnete man ursprünglich die für das antike Rom gängige Form der Sozialität - die Beziehung zwischen dem pater familias (dem Familienoberhaupt) und dem client, einem Fremden, der in die Familie aufgenommen wurde (Beletskii, Kulikova, 2011, S. 8,9). Mitte des 20. Jahrhunderts wurde das Konzept der Patron-Klient-Beziehung nach einigen neueren Veröffentlichungen umgedeutet und als nächste Annäherung der Forschung an die Problematik in andere Untersuchungsfelder übertragen, z.B. in die Pädagogik (Giesinger, 2005). Die sprachliche Dimension des Phänomens wurde zum Gegenstand der Analyse in der medizinischen Kommunikation. Der Paternalismus erscheint dabei als eines der Modelle der Beziehungen zwischen Arzt und Patient und wird durch die Eigenschaften seiner interaktionalen Entfaltung charakterisiert (Koerfer, 2008).
In Anlehnung an die Auffassung des Paternalismus von G. Dworkin lassen sich die Teilnehmer, Ziele und die allgemeine Sequenzstruktur einer paternalistischen Interaktion erkennen. "Paternalism is the interference of a state or an individual with another person, against their will, and justified by a claim that the person interfered with will be better off or protected from harm" (Dvorkin 2005).

Aus diskursanalytischer Perspektive wurde die Theorie des kommunikativen Paternalismus als Ergebnis von wissenschaftlichen Untersuchungen (im linguistischen Bereich) am Institut für Philologie und Sprachkommunikation der Sibirischen Föderalen Universität erarbeitet (Beletskii, Kulikova, 2010, 2011). Im Rahmen dieses kommunikationswissenschaftlichen Ansatzes wurde Paternalismus durch seine diskursiven Praktiken auf Makro- und Mikroebene beschrieben.

Als nächste Herangehensweise in Bezug auf daspaternalistischeKonzept, wasziemlichrelevant auch für die Agenda heutiger gesellschaftlicher Prozesse wäre, sind didaktisch-methodische Ansätze und Curricula im Feld „Migration und Bildung“ anzuführen. Diesem aktuellen Thema war z. B. die Tagung „Paternalismus in der sprachbezogenen Erwachsenenbildung“ im Kontext der Migrationsgesellschaft gewidmet (Wien, 2012), wo u. a. die Forschungs- und Entwicklungsprojekte DaZ („Deutsch als Zweitsprache-Kurse als Raum sprachlicher Ermächtigung") vorgestellt und reflektiert wurden.

\section{Problemstellung und Diskussion}

Nach dem knappen Überblick über die Ausgangsgrundlagen des Untersuchungsgegenstandes möchte ich auf das hier vertretene Konzept des interkulturellen Paternalismus eingehen. Mit diesem Begriff (gemeint vor 
allem als kommunikatives Phänomen) wird auf eine gesellschaftliche Konfiguration aufmerksam gemacht, die das Sprechen und Handeln in dem multikulturellen Kontext und in der Migrationsgesellschaft prägt. Im Sinne einer solchen Dimension des Paternalismus handelt es sich um (diskursive) Eingriffe in die Autonomie des kulturell Anderen mit der Absicht seines Schutzes vor seiner vermuteten bzw. zugeschriebenen Irrationalität im Handlungsraum der fremden Kultur (Kulikova, Beletskii 2011). Die Thematisierung der Interkulturalität bildet, wie die Theorie und die empirische Forschung zeigen, den allgemein vermuteten Hintergrund der Irrationalität der Ausländer / Migranten in den meisten Begegnungssituationen aus. Zum verbalen Standardrepertoire gehören dabei solche Sprechereignisse wie Weisungen, Belehrungen, normative Bestimmungen, kritische Äußerungen, indirekte Verbote etc.

Dafür ein Belehrungsbeispiel aus der akademischen Welt. Die Situation ist mit einer ausländischen Gruppe von Erwachsenen verbunden, die in Deutschland eine Fortbildung im Rahmen eines Förderungsprogramms haben. Da sie alle Ausländer sind, wird ihnen automatisch die Irrationalität oder die mangelnde Kompetenz in der fremden Kultur zugeschrieben, was mit Hilfe des folgenden e-mail-Briefs bearbeitet wurde. Dabei wird in diesem Text das Konzept „Sorge“ ausgedrückt, was im Grunde genommen den paternalistischen Diskurs prototypisch konstituiert und entwickelt:

„...Wir werden dann mit den öffentlichen Verkehrsmitteln, d.h. Bus / Bahn zum Hotel Günnewig fahren. Bitte verpacken Sie Ihr Poster so, dass es bei Regen nicht nass wird. Weiterhin erhalten Sie von Paula Ihre Wochenkarte für den öffentlichen Nahverkehr. Bitte verlieren Sie diese nicht! Im Falle eines Verlusts müssen Sie die Kosten für eine neue Karte selbst tragen“.
Ich würde solche Art des Paternalismus, den ich im Rahmen meiner Untersuchung zu typologisieren versuche, als „netten/milden“ Paternalismus bezeichnen.

In Bezug aufRollenverteilungunterscheidet die paternalistische Figuration (bezogen auf das allgemeine Kommunikationsmodell, s. Abb.1) zwischen jenen, denen Bevormundung, Hilfe, Zuwendung, Unterricht und Unterstützung zukommen soll (,die Behandelten“) und denen, die Hilfe und Unterstützung zu geben in der Lage sind („die Behandelnden“). In dieser Logik verstehen sich die Letzteren als „Beschützer“ und als legitime Wächter über Regeln und Handlungsweisen, über das, was gut für „die Behandelten“ ist oder, anders gesagt, auf der einen Seite der Kommunikation steht die Kultur der „Wissenden“, auf der anderen Seite die Kultur der „Unwissenden“ (s. z.B. Menzel, 2000).

Noch eine These hinsichtlich des interkulturellen Paternalismus: Einerseits kann man ihn als eine ziemlich typische Erscheinung in vielen (wenn nicht allen) Kulturen ansehen, andererseits muss man aber davon ausgehen, dass solche Art der Beziehungen wesentlich kulturabhängig ist und dass seine Ausprägung und Grade der Explizitheit in einem hohen Maße bedingt sind durch die Beziehungsstufen der sich begegnenden Kulturen.

Das nächste Beispiel wird hier als ein Fall aus den persönlichen Beobachtungen des Autors des Artikels vorgestellt, das hiermit auch die Frage zum Überlegen aufwirft, nämlich, inwieweit sich die paternalistisch verlaufenden Situationen zwischen einerseits dem Kreis der Eigenen und andererseits dem Kreis der Fremden unterscheiden, konkret etwa zwischen den westlichen (amerikanisch/europäischen) und den östlichen (z.B., russisch-chinesische) Verhaltensweisen. 


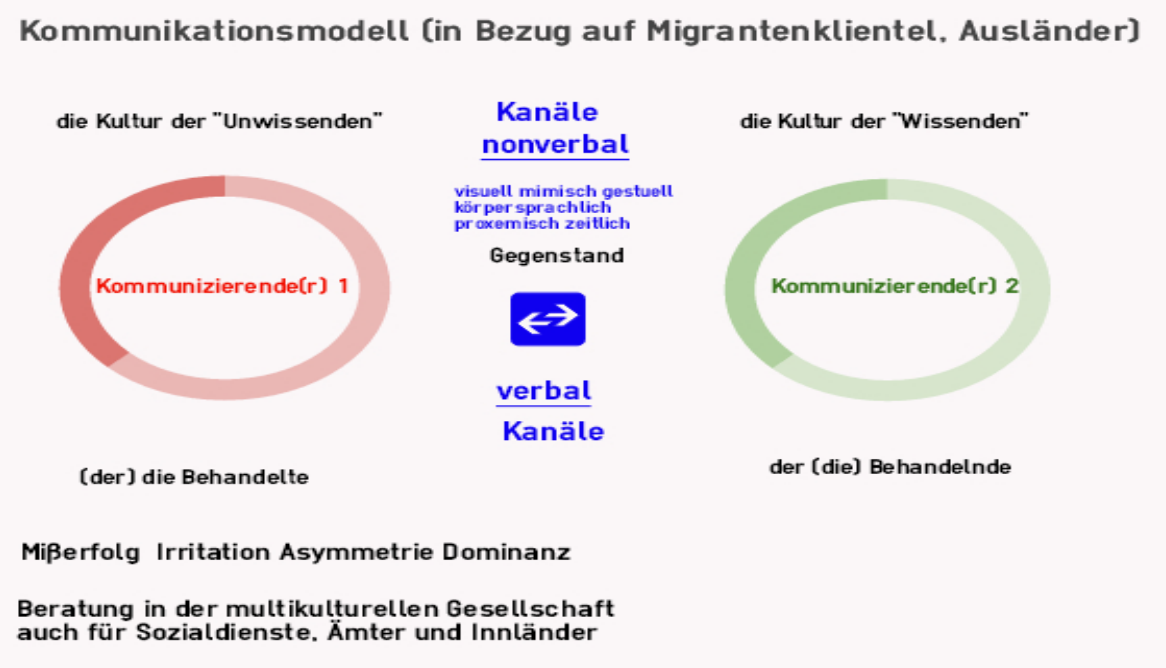

Abbildung 1

\section{Fallbeispiel ,,Spagetti“}

Ein Restaurant in einer deutschen Stadt. An einem hinteren Tisch sitzen drei Chinesen. Zwei Frauen und ein Mann. Eine Frau ist die Ehefrau des Mannes. Am Nebentisch sitzt eine fünfköpfige deutsche Familie. Die Chinesen bestellen Spagetti. Die Kellnerin bringt ihnen zum Essen Löffel und Gabel. Die deutsche Familie schaut mit Interesse zum Nachbartisch hinüber. Die Chinesen beginnen etwas ungeschickt mit Löffel und Gabel zu essen. Sie drehen die Spagetti umständlich auf dem Löffel auf. Die deutsche Frau vom Nebentisch möchte den Chinesen beibringen, wie man richtig Spagetti isst. Sie macht mit einem Wink auf sich aufmerksam, lächelt die Chinesen an und beginnt vorzumachen, wie man Spagetti mit Löffel und Gabel isst. Dem Chinesen ist das augenscheinlich nicht sehr angenehm. Er bleibt höflich, aber an seinem Gesichtsausdruck kann man erkennen, dass es ihm unangenehm ist. Trotzdem lächelt er höflich und dankend der Frau am Nebentisch zu.

Als Kommentar zu der oben vorgestellten realen Situation ist es wichtig, Folgendes hervorzuheben. In der chinesischen Kultur hat das „face“-Konzept eine zentrale Funktion, es reguliert sämtliche sozialen Aktivitäten. Das
Auftreten von "Gesichtsverlust" variiert je nach sozialem Status der Person. Je höher der Status, desto gefährdeter ist das „Gesicht“ der betreffenden Person. Dabei entsteht entsprechend eine solcheFragestellung bzw. Denkansatz: Hatdie Handlung der deutschen Frau (die wohl nur einen guten Ratschlag meinte, aber mit den chinesischen Gepflogenheiten wenig vertraut $\mathrm{zu}$ sein schien) in der beschriebenen Interaktionssituation einen potentiell „gesichtsbedrohenden“ Charakter für den Chinesen? Könnte man sich eine solche Situation auch zwischen zwei Deutschen vorstellen?

Für mich aktualisiert ein solcher Eingriff in die Autonomie (Handlungsfreiheit) der Anderen das paternalistische Modell.

Paternalistische Interaktionsmuster kommen in verschiedenen Bereichen und Modi vor, die in meinem Forschungsvorhaben als Kontexte und Formen hervorgehoben werden. Paternalistisches Handeln kann man also typologisieren. Die Untersuchung differenziert zwischen dem staatlichen, institutionellen (z.B. amtlichen, akademischen, rechtlichen etc.) und dem alltäglichen/privaten Kontext. Manche von den genannten habe ich schon 
interpretiert. Sehr wichtig ist es auch, die staatliche Ebene anzugehen. Die Recherche der einschlägigen Literatur zeigt, dass es eine Reihe von wissenschaftlich-praktischen Publikationen aus dem deutschsprachigen Raum gibt, die das paternalistische Herrschaftsverhältnis im staatlichen Kontext problematisieren. So werden z.B.diepaternalistischenLogiken dessogenannten NIP (Nationalen Integrationsplans) beleuchtet, in dem, nach Meinung des Autors von der Uni Bremen (Ching-Ching Pan, 2012), das Bild der hilfsbedürftigen Frau mit Migrationshintergrund konstruiert wird. Als ausgrenzende und paternalistische Institution wird auch die moderne Schule erwähnt. Im Zusammenhang damit, dass in den letzten Jahren der Anteil der Schüler mit Zuwanderungsgeschichte stark gestiegen ist, diskutiert man die Frage, inwieweit die Lehrkräfte bzw. Schulleitungen die Fähigkeit zu kultursensibler und effektiver Kommunikation mit Schülern und Eltern aus Zuwandererfamilien (was auch für Russland kennzeichnend ist) entwickeln.

Aus diskursanalytischer Perspektive stellt der interkulturelle Paternalismus eine sequenzielle Struktur dar:

- Zuschreiben von Irrationalität oder fehlender Kulturkompetenz als handlungs- / konversationsanalytisch beobachtbarer Prozess.

Die Irrationalität oder mangelnde Autonomie oder mangelnde Kompetenz, die oft durch mangelnde Performanz zu Tage tritt, ist der Grund für den paternalistischen Eingriff. Laut Paternalismis-Forscherin Anne van Aaken fehlen den Menschen in vielen Lebensbereichen klare, stabile und wohlgeformte Präferenzen. Begrenzte Rationalität kann regulative Eingriffe in die Freiheitssphäre der Individuen notwendig erscheinen lassen (Aaken van, 2006). Dabei kann dieser Eingriff in verschiedenen Modalitäten verlaufen und zu (Co)konstruktion, Entzug oder Outsourcing von Autonomie werden.
Den folgenden Textauszug aus meinen Daten würde ich als paternalistisches Interaktionsmuster zur Illustration der ersten Sequenz anbieten:

"Dear....,

due to current events I would like to remind you that you are responsible for keeping your rooms reasonably clean. A cleaning service is provided to you two times a week, but that does not mean that the cleaning services are responsible for cleaning up food that is spread around the apartment or are supposed to do profound and extensive cleaning of huge chaos. You are responsible for keeping your room in proper shape! Please note that if you do not so, you will be charged additional cleaning fees. This information is also important for the time when you will move out of the apartment. When you move out, you are not allowed to leave food, waste, big boxes or anything like this in the room. We have a concept in Germany that is called ,besenrein" and this might be good to know also with regard to interacting with your future German landlords. „Besenrein“ means that you won't have to do a full cleaning of the flat or room yourself, but that you have to empty out the trash, move out all stuff, wash the dishes.

Kind regards and a lovely day to all of you".

Am besten empirisch analysierbar ist natürlich die sprachliche Performanz (situative Verwendung der Sprache). Eingriffe in Kompetenz, die durch mangelnde Performanz verursacht werden, stehen im Zentrum der Aufmerksamkeit im untersuchten Feld.

So habe ich in den Publikationen der österreichischen Migrantin, die das Problem der Sprachbarriere und der Sprachordnungen als Mittel der Hierarchisierung, Machtrelation und Herabsetzung in der amtlichen deutschsprachigen Migrationsgesellschaft betrachtet, genau Folgendes gefunden: 
„Zugleich bemühen sich „gutmeinende Personen" mit uns gebrochen Deutsch zu sprechen, vermutlich in der Annahme, dass es für uns sonst schwer wäre, sie zu verstehen. Andere machen uns „Komplimente“, dass wir schon so gut Deutsch sprechen können ... Die Letzteren wollen uns nicht beleidigen. Die Abwertung und Deklassierung, die in ihren Komplimenten und in ihr Entgegenkommen stecken, sind ihnen gar nicht bewusst und nur schwer nachvollziehbar. Nicht desto weniger sind das für uns Akte der Herabsetzung, mit denen eine dominante Mehrheit versucht, uns unseren Platz zuzuweisen und unser Verhalten zu bestimmen“ (Ivanova, 2012).

- diskursive Bearbeitung/Behandlung der zugeschriebenen Irrationalität, die sich in verschiedenen Sprechhandlungen (Formen), vor allem aber in der (asynchronen), textbasierenden Kommunikation manifestiert.

Dabei versteht man hier unter der diskursiven Bearbeitung einen Komplex von sprachlichkommunikativen Handlungen, die auf die Bewältigung der Irrationalität der Behandelten ausgerichtet ist.

Im monokulturellen Raum gehören dazu z.B. solche Texttypen wie paternalisierende Rechtsnormen: Helmpflicht, Drogenverbot, Sozialversicherungspflichten; Konsumentenschutzbestimmungen, Informationskampagnen u.a. Als ein typisches Beispiel könnte ich dafür folgenden Ausdruck, der ungefähr ähnlich in vielen Kulturen vorkommt, anführen: „Dieser Raum wird in Ihrem Interesse videoüberwacht!“. Das ist eine der verbreiteten Varianten der paternalistischen kommunikativen Episode, der wir Irrationalität der Besucher als Vorannahme entnehmen können, und die ungefähr so lauten kann: Die meisten (alle) sind Diebe!

Im nächsten Beispiel, das auch einem Brief an die Ausländer entnommen ist, schreibt man den Letzteren eine gewisse Irrationalität zu (Irrationalität wird hier situativ konstruiert), und versucht diese diskursiv zu behandeln:

$$
\text { „Liebe ..., }
$$

Vielen Dank auch für eure interessanten Fragen gestern bei der .... und heute bei der .... Eine Bitte an Euch, es wäre schön wenn ihr Smartphone use in den Terminen gering halten könntet, da dieses in Deutschland oft als nicht besonders höflich empfunden wird. Wir verstehen natürlich auch, wenn ihr für Telefonanrufe oder Toilettengänge den Termin kurzfristig verlassen müsst. Es wäre allerdings schön, wenn wir das auf ein Minimum reduzieren könnten. Ständiges rein und raus verursacht sehr viel Unruhe, wie ihr alle sicher selber bestätigen könnt, genauso wie Unterhaltungen im Hintergrund. Vielen Dank!“

Also, vermeintlich auf das Wohl der Ausländer_innen zielend, kann hier das rein paternalistisches Muster als Einschränkung von Selbstbestimmung und -verfügung und - genau wie die Befragten sagen - irritierend und sogar beleidigend wirken.

- Akzeptieren von oder Abneigung gegen Bevormundung und normative Zurichtungen durch geschriebene vs gesprochene Diskurse (als den verbalen oder nonverbalen Umgang mit dem paternalistischen Eingriff).

Falls der Behandelnde die vorgeschlagene Hilfe, d.h. den Paternalismusanspruch nicht akzeptiert, spricht man u.a. von der Entwicklung eines Gegendiskurses, z.B. in Form von Veröffentlichungen. Seitens des Behandelten wäre dann die Rede von neuen Konzepten interkultureller Arbeit und Beratung mit der Migrantenklientel als „Gegendiskurs“ zu Praktiken der Entmündigung und Herabsetzung.

Auffällig in Hinblick auf diese Stufe der paternalistischen Praktiken wären die Muster der öffentlichen Auseinandersetzung bzw. Integrationsdebatte in Deutschland in letzter Zeit, 
die ich hier wissenschaftlich problematisieren möchte.

Mit Hilfe der kommunikativen Mittel (des geschriebenen Diskurses) erfolgt in vielen Orten in Deutschland die Bearbeitung nicht nur vermuteter, sondern auch empirisch erkannter Irrationalität der Flüchtlinge / Migranten. Es handelt sich z.B. um den bekannten Text des Willkommensbriefes eines Bürgermeisters, der sich an die Migrantengäste der Stadt wendet (http://www.hardheim.de/sites/brief.htm):

„Liebe fremde Frau, lieber fremder Mann!

Willkommen in Deutschland, willkommen in Hardheim. Viele von Ihnen haben Schreckliches durchgemacht. Krieg, Lebensgefahr, eine gefährliche Flucht durch die halbe Welt. Das ist nun vorbei. Sie sind jetzt in Deutschland. Deutschland ist ein friedliches Land. Nun liegt es an Ihnen, dass sie nicht fremd bleiben in unserem Land, sondern ein Zusammenleben zwischen Flüchtlingen und Einwohnern erleichtert wird. Eine Bitte zu Beginn: Lernen sie so schnell wie möglich die deutsche Sprache, damit wir uns verständigen können und auch sie ihre Bedürfnisse zum Ausdruck bringen können. In Deutschland leben die Menschen mit vielen Freiheiten nebeneinander und miteinander: Es gilt Religionsfreiheit für alle. Frauen dürfen ein selbstbestimmtes Leben führen und haben dieselben Rechte wie die Männer. Man behandelt Frauen mit Respekt. In Deutschland respektiert man das Eigentum der anderen. Man betritt kein Privatgrundstück, keine Gärten, Scheunen und andere Gebäude und erntet auch kein Obst und Gemüse, das einem nicht gehört. Deutschland ist ein sauberes Land und das soll es auch bleiben! Den Müll oder Abfall entsorgt man in dafür vorgesehenen Mülltonnen oder Abfalleimer. Wenn man unterwegs ist, nimmt man seinen Müll mit zum nächsten Mülleimer und wirft ihn nicht einfach weg. In Deutschland bezahlt man erst die Ware im Supermarkt, bevor man sie öffnet. In Deutschland wird Wasser zum Kochen, Waschen, Putzen verwendet. Auch wird es hier für die Toilettenspülungen benutzt. Es gibt bei uns öffentliche Toiletten, die für jeden zugänglich sind. Wenn man solche Toiletten benutzt, ist es hier zu Lande üblich, diese sauber zu hinterlassen. In Deutschland gilt ab 22.00 Uhr die Nachtruhe. Nach 22.00 Uhr verhält man sich dementsprechend ruhig, um seine Mitmenschen nicht zu stören. Auch für Fahrradfahrer gibt es bei uns Regeln, um selbst sicher zu fahren, aber auch keine anderen zu gefährden. (Nicht auf Gehwegen fahren, nicht zu dritt ein Rad benutzen, kaputte Bremsen reparieren und nicht mit den Füßen bremsen). Fußgänger benutzen bei uns die Fußwege oder gehen, wenn keiner vorhanden, hintereinander am Straßenrand, nicht auf der Straße und schon gar nicht nebeneinander. Unsere Notdurft verrichten wir ausschließlich auf Toiletten, nicht in Gärten und Parks, auch nicht an Hecken und hinter Büschen. Mädchen und junge Frauen fühlen sich durch Ansprache und Erbitte von Handy-Nr. und facebookKontakt belästigt. Bitte dieses deshalb nicht tun! Auch wenn die Situation für sie und auch für uns sehr beengt und nicht einfach ist, möchten wir sie daran erinnern, dass wir sie hier bedingungslos aufgenommen haben. Wir bitten sie deshalb, diese Aufnahme wert zu schätzen und diese Regeln zu beachten, dann wird ein gemeinsames Miteinander für alle möglich sein".

Bezogen auf das hier behandelte Konzept haben wir in diesem Beispiel mit der diskursiven Bearbeitung der zugeschriebenen Irrationalität oder der mangelnden konventionellen Kompetenz im Rahmen der paternalistisch verlaufenden Situationen zu tun. Man könnte solche Form des interkulturellen Paternalismus entweder legitimen verpflichtenden Paternalismus oder staatlich- 
berechtigten unumgänglichen Paternalismus nennen.

Dabei entstand gleichzeitig im deutschen Medienraum als Reaktion auf den Paternalismusanspruch des bekannt gewordenen „Willkommensbriefs“" ein so genannter Gegendiskurs. Es ist analytisch sinnvoll, die von manchen Zeitungen gewählte Form der Abneigung hier anzuführen (http://www. refugeeguide.de/de/):

"Die Regeln transportieren Vorurteile und Ressentiments. Flüchtlinge sind Diebe, schmutzig und baggern allesamt Mädchen an - das bleibt nach der Lektüre des Leitfadens hängen. Man weiß, dass einige der Hinweise als überheblich oder abwertend empfunden werden können."

Wie es anders gehen kann, wie auch ein respektvoller Ton gelingen kann, ohne dass die Aussagen an Deutlichkeit verlieren, zeigt ein umfangreicher Guide für Flüchtlinge, den Ehrenamtliche verschiedener kultureller Herkunft und aus ganz Deutschland verfasst haben. Darin steht zum Beispiel:

„Homosexualität ist Normalität in Deutschland (ein ehemaliger Außenminister war offen schwul). Homosexuelle Partnerschaften können legal und der Ehe ähnlich registriert werden."

„Privatsphäre ist Menschen in Deutschland wichtig. Das kann manchmal distanziert wirken. Es ist vollkommen normal, stundenlang im Zug oder im Restaurant neben Fremden zu sitzen und nur „Guten Tag" oder ,Auf Wiedersehen" zu sagen. Genauso ziehen es einige Leute vor, alleine zu sitzen, zum Beispiel in öffentlichen Verkehrsmitteln."

„Der Umgang mit der Bürokratie istmanchmal sehrkomplexund kannineffizient wirken. Bürokratie braucht Zeit und folgt standardisierten Abläufen. Das kann frustrierend sein."

Im Unterschied zum Hardheimer Leitfaden benennt auch dieser Guide klare Regeln und
Gesetze, aber er gibt Flüchtlingen auch echte Hilfestellungen - er beschreibt zum Beispiel, welche Einkaufsmöglichkeiten es in Deutschland gibt.

Sie haben sich Gedanken gemacht darüber, wie ihr Leitfaden auf Flüchtlinge wirken könnte, schreiben die Autoren. „Dies wurde bei der Erstellung kontinuierlich kritisch hinterfragt und reflektiert. Um dieser Unsicherheit zu begegnen, wurde diese Orientierungshilfe in enger Zusammenarbeit mit Menschen aus verschiedensten Ländern verfasst."

In Anlehnung an das Höflichkeitskonzept (Brown, Levinson, 1987) lohnt es sich in diesem Fall von der Art des positiven Paternalismus $\mathrm{zu}$ sprechen, mit den Indikatoren, die Einverständnis signalisieren, Strategien der Sympathiebekundung, der Annäherung und der „in-group-Markierung“.

\section{Zusammenfassung}

In diesem Artikel hat der Autor versucht, paternalistisches Handeln in der modernen Migrationsgesellschaft als interkulturelles Phänomen aus diskursanalytischer Sicht zu interpretieren. Es stellte sich heraus, dass die diskursive Behandlung der zugeschriebenen Irrationalität oder der mangelnden konventionellen Kompetenzen im Rahmen der paternalistisch verlaufenden Situationen eine Reihe von Praktiken voraussetzt. Es werden auch Kontexte (institutionell, alltäglich, staatlich, privat) des interkulturellen Paternalismus behandelt und seine Formen typologisiert (s. Abb.2). Bezogen auf dieses Konzept kann man den Mechanismus der Entwicklung der paternalistischen Praktiken (vom sprachlich-kommunikativen Standpunkt aus) aufdecken. Dabei handelt es sich entweder um Abneigung, die paternalistischen Diskurse als Legitimation von Herrschaft in der Zuwanderungsgesellschaft nicht zu tolerieren, 


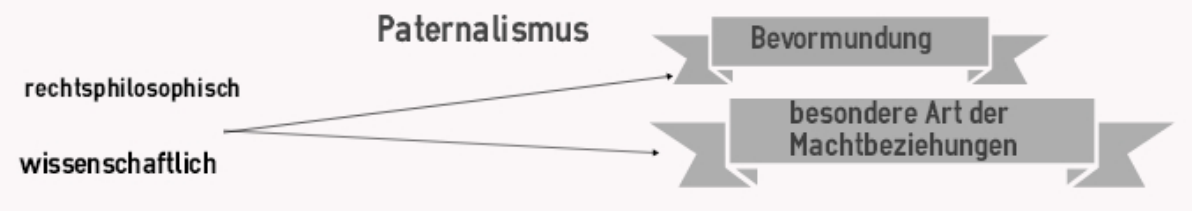

\section{Interkultureller Paternalismus (Migrationsgesellschaft)}

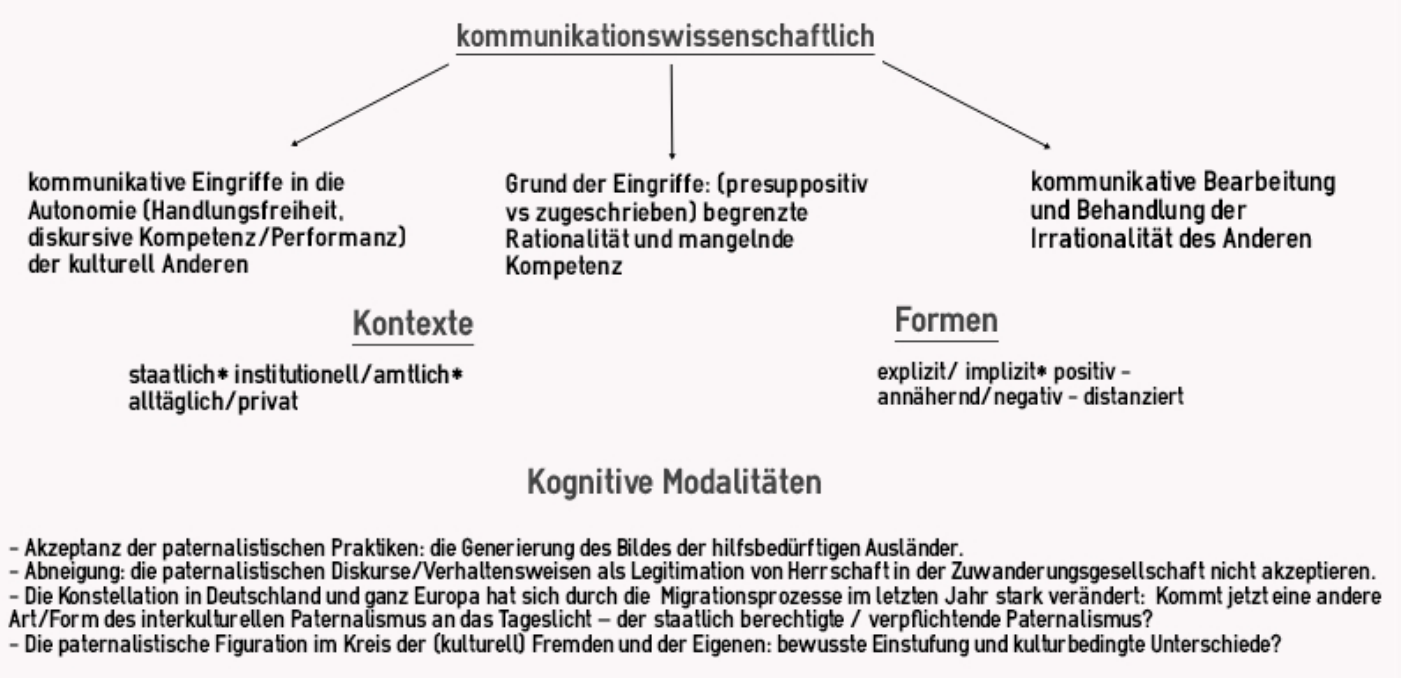

Abbildung 2

interkulturell kompetent und kultursensibel zu handeln, oder um Akzeptanz: die Generierung des Bildes der ausnahmsweise hilfsbedürftigen Ausländer. Das heißt, aus der sozialen Praxis ergeben sich ganz bestimmte Bedarfe, welche neue Konzepte interkultureller Arbeit und Beratung hervorrufen.

Eine Zitat zum Schluss: „Es gibt Kriegsgeschichte, Kriegskunde, Waffenkunde; Toleranzkundeinauchnurentferntvergleichbarem Ausmaß gibt es nicht (Mitscherlich)“. Das
Schlüsselwort Toleranz habe ich hervorgehoben, weil ich es unter anderem als Voraussetzung sehe, damit die Kontaktsituationen zwischen Inländern und Ausländern in verschiedenen Verhaltensbereichen nicht zu Disparitäten und Irritationen führen. Im Grunde genommen wirkt der paternalistische Zwang kontraproduktiv und gerade das tolerierende Verstehen und Anerkennen der Differenz sollte in der modernen Migrationsgesellschaft im Vordergrund stehen.

\section{Literaturangaben}

Aaken van, A. (2006). Begrenzte Rationalität und Paternalismusgefahr: Das Prinzip des schonendsten Paternalismus, In Paternalismus und Recht. Tübingen: Mohr Siebeck, 109-145.

Beletskii, S. (2010). Paternalism v instituthional'noi kommunikatsii: diskursivnye praktiki, strategii i zhanry [Paternalism in Institutional Communication: Discursive Practices, Strategies and Genres], Ph.D. Krasnojarsk, 176 p.

Beletskii, S., Kulikova, L. (2011). Paternalism v instituthional'noi kommunikatsii [Paternalism in Institutional Communication]. Moscow, Flinta, Nauka, 184 p. 
Brown P., Levinson, S. (1987). Politeness: Some Universals in Language Usage. Cambridge: Cambridge Univ. Press, 333 p.

Ching-Ching, Pan (2012). Der Paternalismus im Nationalen Integrationsplan am Beispiel des Geschlechterrollenverständnisses. Beiträge der Tagung „Paternalismus in der sprachbezogenen Erwachsenenbildung" im Kontext der Migrationsgesellschaft gewidmet. Wien.

Dvorkin, G. (2005). Paternalism, In The Stanford Encyclopedia of Philosophy, available at: http:// plato.stanford.edu/archives/win2005/entries/paternalism/ (accessed 5 August 2016)

Giesinger, J. (2005). Pädagogischer Paternalismus. Eine ethische Rechtfertigung: Dissertation. Zürich. Available at: http://www.dissertationen.unizh.ch/2005/giesinger/abstract.html (accessed 5 August 2016)

Heiko, U.Z. (2006). Paternalismus. Fallstudien zur Genese des Begriffs. Freiburg, Alber, 250 S.

Hilfestellung und Leitfaden für Flüchtlinge (2015). Available at: http://www.hardheim.de/sites/ brief.htm (accessed 5 August 2016)

Ivanova, M. (2012). Welche Handlungsmöglichkeiten haben Migrant_innen in Österreich paternalistische Praktiken und Diskurse im Kontext von Sprachen - Ordnungen zu begegnen. Beiträge der Tagung „Paternalismus in der sprachbezogenen Erwachsenenbildung“ im Kontext der Migrationsgesellschaft gewidmet. Wien.

Koerfer, A. (2008). Training und Prüfung kommunikativer Kompetenz. Aus- und Fortbildungskonzepte zur ärztlichen Gesprächsführung, In Gesprächsforschung, 9, 34-78.

Menzel,P.AusländervorGericht.MißverständnisseinGerichtsverhandlungenzwischenAusländern und Experten. Eine ethnopsychologische Studie im Rahmen der interkulturellen Kommunikation, In Mit der Differenz leben. Europäische Ethnologie und Interkulturelle Kommunikation, München, Hgg. Roth K., 2000, 166.

Mitscherlich, A. (1983). Wie ich mir - so ich dir. Zur Psychologie der Toleranz, In Gesammelte Schriften, Band 5, Sozialpsychologie, 3, 410-428.

Refugee Guide Online. Available at: http://www.refugeeguide.de/ (accessed 5 August 2016)

\section{Дискурсивные практики}

межкультурного патернализма

в современных миграционных обществах

(на примере немецкоязычного пространства)

Л.В. Куликова

Сибирский федеральный университет Россия, 660041, Красноярск, пр. Свободный, 79

В статье раскрывается понятие межскультурного патернализма и его дискурсивная реализаиия в мультикультурном контексте современных миграчионных обществ (на коммуникативноязыковом материале немецкоязычного пространства).

На основе теоретических обобщений и собранных эмпирических данных автор рассматривает возможности типологизаџии дискурсивных практик патернализма в корреляции с институци- 
ональными и неинституцчинальными сферами его бытования, прагматикой межкультурных ситуаџий и дифференцииачией ролевых позищий коммуникантов. В исследовании выявляется суть дискурсивной обработки патерналистских действий и на примере авторских кейсов подробно описываются способы дискурсивной обработки в ситуаџиях взаимодействия актантов с разной культурной конвенциональностью. Фокусируется внимание на необходимости изменения поведенческих паттернов в структурах власти, миграционных службах, образовательных и общественных организациях в условиях новых сощиальных реальностей.

Основными методами проведенного мини-исследования являются включенное наблюдение, интервьюирование, кейс-методика, систематизирующий анализ профильной научной литературы и изданий немецкоязычной прессы, мониторинг медийного пространства.

Ключевые слова: патерналистский дискурс в мультикультурном обществе, контексты и типь/ формы межкультурного патернализма, дискурсивная обработка патерналистских практик, этапь дискурсивной обработки.

Научная специиальость: 10.00.00 - филологические науки. 